\title{
A PARALLEL ALGORITHM FOR TOPOLOGY OPTIMIZATION
}

\author{
Roy Johanson* \\ Panos Papalambros \\ Newton Mack \\ Noboru Kikuchi \\ The University of Michigan \\ Design Laboratory \\ Department of Mechanical Engineering \\ and Applied Mechanics \\ Ann Arbor, MI 48109-2125
}

\begin{abstract}
We demonstrate an efficient algorithm for the optimum topology design of continuum structures using parallel computers. The homogenization method provides a rigorous method for determining the optimum topology of a structure subject to specified loads and boundary conditions. This paper describes our efforts to improve the computational efficiency of the method by developing parallel algorithms for the structural analysis and optimization procedures.
\end{abstract}

\section{Background}

Homogenization Method

Structural optimization, has been an active area of research since the early 1970s; see for example [Botkin ${ }^{5}$; Fleury ${ }^{8}$; Bennet and Botkin $^{4}$; Kikuchi ${ }^{9}$; Esping ${ }^{7}$ ]. The two basic optimization problems addressed in structural optimization have been sizing and shape optimization. In sizing optimization, variables define local geometric characteristics such as height, width, thickness, and radius of specific portions of the structure. A typical design task is to find the minimum weight shell structure to withstand applied thermo-mechanical loads. In shape optimization, the optimum shape of a structure is sought by varying the boundary shape defined by an appropriate spline function, with the design variables defined in a function form.
In most design problems, the topology of a structure is not known a priori. Frequently, topology is related to the number of holes in a structure. If the topology is fixed, the configuration is defined easily by spline functions. Significant difficulties are encountered when the topology of a structure must be designed, since its representation with spline-type functions is unwieldy. As a result, design problems involving both shape and topology have not been solved satisfactorily. Several approaches to the topology optimization problem have been proposed: see, for example, Rozvany ${ }^{13}$, and the proceedings of a recent NATO ASI (Bendsoe, 1992) ${ }^{3}$. The homogenization method, described by Bendsoe and Kikuchi (Bendsoe, 1988) ${ }^{2}$ is unique in that it yields the optimal shape and topology at a macro- and micro-level of description.

The necessity of topological design in addition to size and shape design is widely recognized by structural engineers. If topological changes are not allowed, size and shape optimization procedures can improve a design by approximately $5 \sim 15 \%$. Topological modifications can often yield $30 \sim 50 \%$ improvement. An example illustrates this. The beam in Figure 1 is subjected to a bending moment. A hollow beam is more effective than a solid beam. For the same amount of material, the beam design shown at the bottom of the Figure, which involves topological changes, is better than the one at the top, which can be derived by shape optimization. 

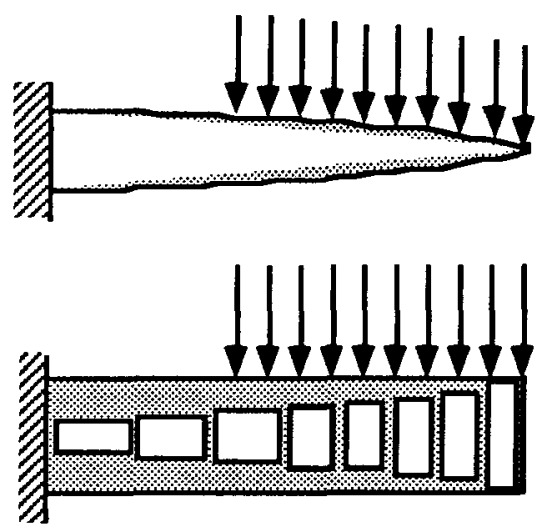

Figure 1: Shape Design and Topology Change of a Structure

The homogenization method is based on the above observation. The topology and shape problem is formulated as a new optimization problem involving material distribution. Given a solid with a prescribed volume, we generate microscale voids in design domains where a solid structure is not required for supporting loads. Therefore, instead of designing the shape and physical dimensions of the cross section of a structure, we generate infinitely many microscale voids within the configuration wherever the stress is small. If a portion in the domain is highly stressed the homogenization method prevents the creation of microscale holes, and that portion remains solid. Furthermore, the orientation of a non-circular void has a significant effect on the overall material response. Therefore, in the new optimization problem, the design variables are the density of microscale voids and their orientation over a specified domain. By removing material completely from portions of the domain densely packed with voids, the optimum shape of the structure is identified, while its topology is determined by accounting for the number of "global" holes.

This intuitive method of "shaping and drilling" a structure is based on the theory of homogenization -- a mathematically rigorous method developed in the mid-1970s for the study of mechanics of composite materials. Most composite materials possess a fine scale microstructure composed of fibers, whiskers, inclusions, and matrices. Applied mathematicians in France, Italy, and the former Soviet Union [Lurie and Cherakev ${ }^{11}, \operatorname{Tartar}^{14}$ ] developed the homogenization theory to derive the constitutive equation of a composite material, i.e., to evaluate the average stress-strain relation of the structure. Since we are interested in generating infinitely many microscale holes to form a possibly perforated structure, the stress analysis of such a structure requires the derivation of an equivalent effective average stress-strain relation. A homogenization approach enables the design of topology and shape without using spline functions. Difficulties in geometric modeling are avoided, and stress analysis iterations are performed on a fixed finite element mesh.

\section{Mathematical Formulation}

We can formulate a generalized topology optimization problem by introducing microstructural perforations into the structure, and then minimizing the mean compliance subject to a constraint on the total volume of material used. Formally,

$$
\underset{a, b, a \text { and } \theta}{\operatorname{Minimize}} \sum_{i} f_{i} u_{i} d \Omega+\sum_{i} \int_{\Gamma_{1}} t_{i} u_{i} d \Gamma
$$

subject to equilibrium equations, and

$$
\int_{\Omega}(1-a b) d \Omega \leq \Omega_{s}
$$

Here, $u$ is the vector of virtual displacements, $f$ is the applied body force, $t$ is the applied traction on the boundary $\Gamma_{t}, i$ is the number of finite elements used to discretize the structure, and $\Omega_{\mathrm{S}}$ is the total volume of solid material forming the porous structure. The microstructural model used in this method is shown in Figure 2, and possesses three design variables per element: the void dimensions $a$ and $b$, and the void orientation angle $\theta$. The design variables $a$ and $b$ are restricted to values between 0 and 1.

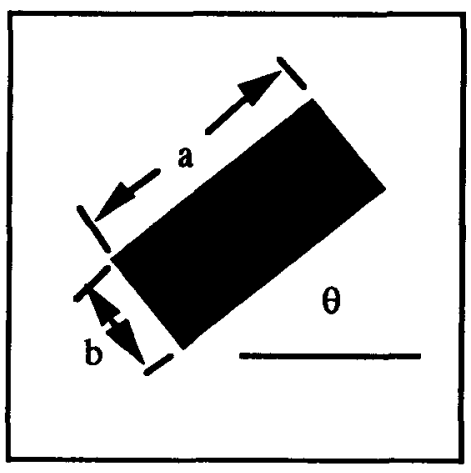

Figure 2: Element Microstructural Model 
The equilibrium equation and its associated loading and support conditions, i.e., the structural analysis problem, are solved using the finite element method. The domain for stress analysis is the initial design domain. This initial domain is then discretized into finite elements, with the design variables $a, b$, and $\theta$ for each element evaluated at the centroid. The number of design variables for the present problem is quite large, and it is difficult to apply standard mathematical programming techniques. The optimization method used for this problem is a simple resizing scheme based on the optimality conditions. For details on the optimization method see Bendsoe 2 .

We have extended this basic formulation to 2-D, 3-D and shell structures. Additionally, we have extended the formulation to allow topology optimization to create structures with desired natural frequency constraints.

\section{Parallel Implementation}

Applications of parallel computing to structural analysis and optimization have been steadily increasing. Examples of some recent work include El-Sayed and Hsuing ${ }^{6}$ and Xicheng and Guixu ${ }^{15}$. In this study, we explore the parallelization of the 2-D homogenization algorithm described above. The parallel algorithm described here is implemented on a Kendall Square Research KSR1. The KSR1 is a shared-memory parallel computer, and the particular machine used in this research has 64 processors.

The major change in the topolgy optimization algorithm was the implementation of a efficient method for the parallel solution of the large system of equations arising in the finite element model used for structural analysis. A considerable portion of the computation time is spent in evaluating the finite element model at every iteration. The algorithm used for the solution of this linear system is a Cholesky decomposition, modified for implementation on a parallel computer. The method implemented here is conceptually similar to one proposed by Zmijewski and Gilbert ${ }^{19}$.

For efficient parallel computing, all tasks that consume significant computation time must be divided between processors, and overhead between processors should be minimized. All procedures were inlined, and most vector operations were parallelized.

\section{Examples and Results}

We consider two classes of examples: timing examples, to demonstrate the efficacy of the parallel implementation, and design examples to demonstrate the utility of the homogenization method for structural layout design.

\section{Timing Studies}

A simple bracket design problem was used to test the parallel topology optimization algorithm described above. This problem was solved several times, with increasingly fine mesh density. Figure 3 shows the effect of increasing the number of processors used in the solution of a small (100 element) topology optimization problem. Clearly, for small problems, the overhead encountered in the allocation of processors and the sharing of data overwhelms any computational advantage that is realized through parallelization.

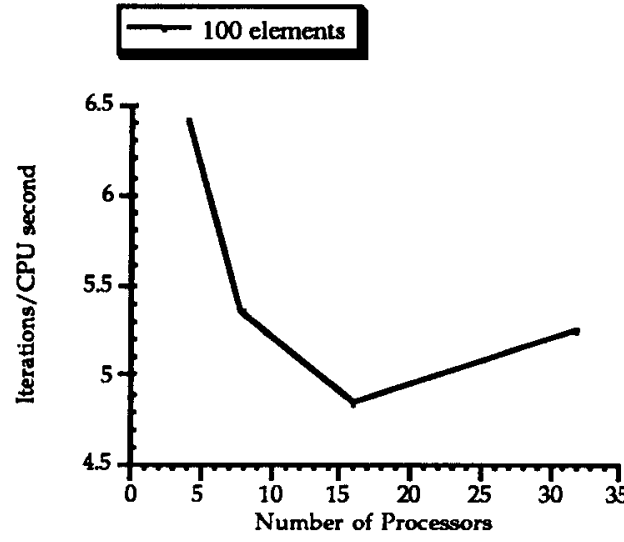

Figure 3: CPU time as a function of Allocated Processors -- small problem.

For a larger problem (2000 elements) the effect of increased processor utilization becomes more profound. Figure 4 shows the observed performance of the parallelized homogenization algorithm on a fairly large topology optimization problem. As the size of the problem increases, the speed advantages realized by parallelization become more pronounced. More efficient parallel assembly of the finite element stiffness matrix at every iteration and processor allocation to reduce communication overhead will increase the overall observed speed of the method as well as enhance 
the effects of parallelization. The observed speedup in processing time, while encouraging, is not as good as might be expected as the number of processors used increases. This seems to indicate that the inter-processor overhead in our implementation still consumes a significant portion of the computation time.
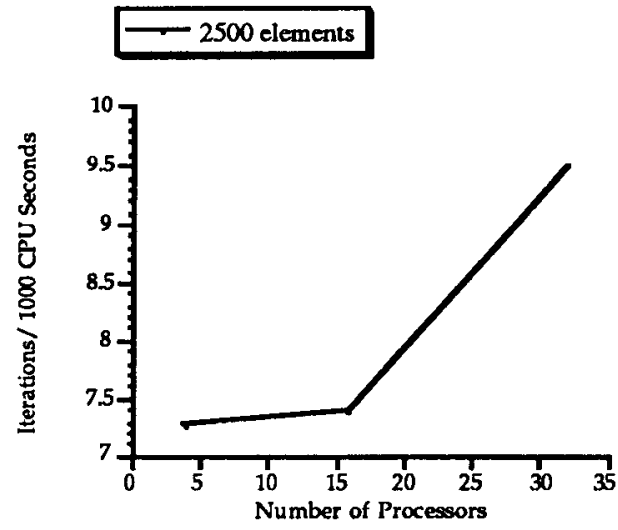

Figure 4: CPU time as a function of Allocated Processors -- large problem.

\section{Design Example}

We offer one example to demonstrate the utility of such a topology optimization tool. Consider the road sign support design problem outlined in Figure 5. The objective of this problem is to design a structure within the limitations of the design domain which possesses minimum compliance when subjected to the loading and boundary conditions shown.

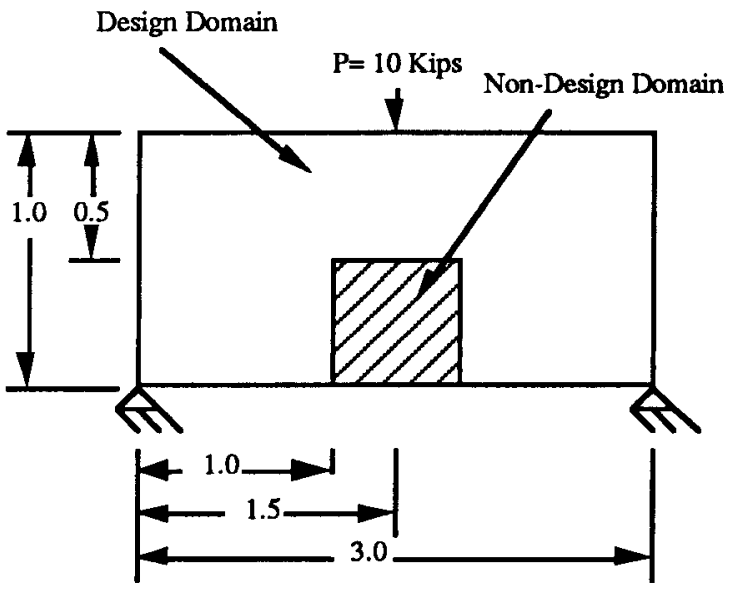

Figure 5: Design Domain and Loading Conditions for Road Sign Support Problem

Two cases are considered: designs which allow structure in the area designated as the nondesign domain, and designs restricted to regions outside the non-design domain. The initial design domain is discretized with 1200 elements, and the topology optimization problem is solved twice: once with material distribution allowed everywhere in the initial design domain, and once with the material distribution restricted to be outside of the non-designable region shown above. Figures 6 and 7 show the results of the homogenization procedure. Dark areas on the Figures indicate regions of solid material, i.e., areas where the size of the microscale voids has been reduced to zero. White areas indicate regions where material is not required in the optimal structure. Areas of intermediate grayscale shading indicate regions of composite structure where the structure possesses microstructure. In many cases, these areas can be considered to be regions which possess different directional stiffness characteristics. Depending on the problem application and the manufacturing technology available, these intermediate regions may either be interpreted as representing isotropic material or as representing composite structure. This layout design is then ready to be subjected to detail sizing and shape optimization, where additional constraints on local measures, such as stresses, are considered.

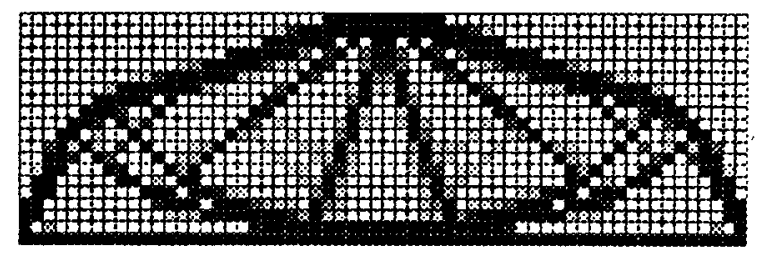

Figure 6: Optimum Road Sign Support

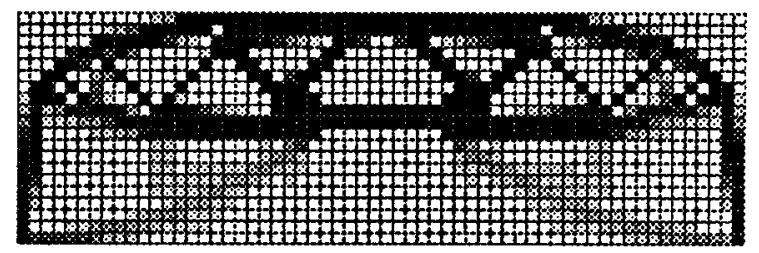

Figure 7: Optimum Topology for Road Sign Support with Non-Designable Region

The effect of introducing the geometric design constraint is striking. The resultant structure bears a strong resemblance to overhead road sign structures currently in use. The only inputs from the designer are the loads, boundary conditions, and the specification of the design domain.

Comparison of the solution time for this problem between serial and parallel 
implementations shows a modest improvement as the number of processors used is increased.

\section{IV: Conclusions}

A parallel algorithm for the optimal topology design of continuum structures has been described. Observed performance improvements are significant, but still modest for small problems. The computational speed improvements due to parallelization become more pronounced as the size of the problem increases. Clearly, use of efficient parallel algorithms will allow the consideration of larger classes of topology optimization problems. Development of efficient algorithms on parallel computers requires somewhat different programming techniques than those used to program on serial computers.

\section{Acknowledgments}

Computational facilities for this work were partially provided by the U-M Center for Parallel Computing under NSF grant CDA-9214296. This support is gratefully acknowledged. Thanks also to Mr. Stephen Ambo for suggesting the road sign support design example.

\section{References}

1. Anderson, E. et. al. LAPACK Users Guide, SIAM, 1991.

2. Bendsoe, P.M., and Kikuchi, N., "Generating Optimal Topologies in Structural Design Using a Homogenization Method", Comp. Meth. Appl. Mech. Eng., Vol 71, 197-224, 1988.

3. Bendsoe, M.P. and C.A. Mota Soares, eds., Topology Design of Structures, NATO ASI, Kluwer, 1992.

4. Bennet, J.A and Botkin, M.E., The Optimum Shape: Automated Structural Design, Plenum Press, New York 1986.

5. Botkin, M.E. and Bennet, J.A., "Shape Optimization of Three Dimensional Folded Plate Structures", AIAA J,, 23(11) 1804$1810,1985$.
6. El-Sayed, M. E. and C.K. Hsiung, "Optimal Structural Design with Parallel Finite Element Analysis" Computers and Structures, 40 (6) , pp. 1469-1474, 1991.

7. Esping, B.J.D, The OASIS Structural Optimization System, Report No. 85-3, The Royal Institute of Technology, Stockholm, 1985.

8. Fleury, C., and Braibant, V., "Structural Optimization: A New Dual Method Using Mixed Variable", Int. J Num. Methods in Eng, Vol 23, 409-428, 1986.

9. Kikuchi, N., Chung, K.Y., Torigaki, T., and Taylor, J.E., "Adaptive Finite Element Methods for Shape Optimization of Linearly Elastic Structures", Comp. Meth. Appl. Mech. Eng., Vol 57, 67-91, 1986.

10. Kohn, R.V., and Strang, G., "Optimal Design and Relaxation of Variational Problems", Comm. Pure Appl. Math., Vol 39, 1-25 (Part I) 139-182 (Part II) 353-377 (Part III), 1986.

11. Lurie, K.A, and Cherakev, A.V., "Exact estimates of conductivity of composites formed by two isotropically conducting media taken in prescribed proportion", Proc. Royal Soc. Edinburgh, 99A, 71-78, 1984.

12. Mota Soares, C.A., (ed.) "Computer Aided Optimal Design: Structural and Mechanical Systems" Springer Verlag, Berlin, 1987.

13. Rozvany, G. and M. Zhou, Qptimality Criteria Methods for Large Structural Systems, Research Report, UniversitatGesamthochschule-Essen, March, 1993.

14. Tartar, L., "Estimation de coefficients homogeneises, Springer Lecture Notes in Mathematics, Berlin 364-373, 1977.

15. Xicheng, W. and M. Guixu, "A Parallel Iterative Algorithm for Structural Optimization," Comp Methods Appl. Mech. Engr, 96 (1992), pp. 25-32.

16. Zmijewski, E. and J.R. Gilbert, "A parallel algorithm for sparse symbolic Cholesky factorization on a multiprocessor," Parallel Computing 7 (1988) pp. 199-210. 